XXVII.

\title{
Ueber die physiologische Wertmessung des Digitalysats.
}

\author{
Von
}

C. Focke (Düsseldorf).

(Mit 1 Diagramm im 'Text.)

Im Jahre 1913 waren von mir drei längere Reihen von Wertmessungen mitgeteilt worden, die ich an Temporarien mit Gitalin (C. F. Boehringer und Söhne), mit den Fol. Dig. titr. (Cäsar und Loretz) und mit Digitalinum verum (C. F. Boehringer \& Söhne) durchgeführt hatte ${ }^{1}$ ). Hierbei waren verschiedene lösungsverhältnisse, vom stärksten bis zum schwächsten, und gleichzeitig verschiedene Injektionsmengen angewendet worden, von $1 / 25$ des Froschgewichts (F. G.) herab bis zu den kleinsten, eben noch wirksamen Mengen. Im Jahre 1914 hatte ich dann eine ähnliche Reihe angefügt, die das g-Strophanthin (E. Merck) betraf ${ }^{2}$ ). Aus diesen systematischen Untersuchungen war u. a. wieder hervorgegangen, dass zur physiologischen Wertmessung der Blätter meine bisherige Prüfungsart als "Methode der mittleren Dosen" die geeignetste ist. Aber zur Messung der Reinpräparate hatte sich diese Methode als nicht ausreichend erwiesen; bei ihnen ist eine Feststellung der noch eben wirksamen Mindestdosen nötig.

Es war meine Absicht, nach den Strophanthinversuchen auch möglichst bald das deutsche Digitalis-Dialysat, das Digitalysatum (J. Bürger, Wernigerode am Harz) einer solchen Durchprüfung zu unterziehen. Denn bei seiner Wertmessung hatten sich mir in jedem Jahre gewisse Schwierigkeiten ergeben, infolge deren eine Digitalysat-Messung immer mehr Zeit und Mühe beanspruchte als eine Blättermessung, ohne dass mir die Ursache dieses Unterschiedes erkennbar war.

Ich lührte nun die systematische Prüfung des Digitalysats im Januar und Februar 1914 bis auf einige Lücken durch. Deren Ausfüllung und die Bearbeitung der Befunde wurde aber durch den Krieg verschoben und konnte erst jetzt beendet werden.

Im Folgenden sollen zuerst die Befunde dargelegt, dąnn einige Fragen erörtert werden.

1) Diese Zeitschr. 14. Bd., S. 262-309.

2) Ebenda, 16. Bd., S. 443-466. 


\section{Schematische Prüfung einer Digitalysatprobe, die an Wirkungswert stärker ist als das normale Digitalysat.}

Zur Prüfung wählte ich die aus der Ernte von 1913 stammende grössere Probe IX, deren Wirkungswert merklich höher war als der des normalen Digitalysats. Zwar sollte das normale Digitalysat eigentlich im Mittelpunkt der Untersuchung stehen; aber wenn ich dieses benutzt hätte, so hätte ich es zur vergleichenden Prüfung einer stärkeren Konzentration eindunsten müssen, was ich vermeiden wollte. Die Probe IX war glyzerinfrei; sie enthielt wie das normale Digitalysat angeblich $15 \mathrm{pCt}$. Alkohol und hatte das spezifische Gewicht 1,023. Die weitere Zubereitung geschah so, dass bei dem unverdünnten Präparat $1: 1$ etwa ein Drittel des Alkohols abgedunstet und mit Ringer-Lösung ergänzt wurde. Zur Herstellung der übrigen Lösungen $1: 2$ bis $1: 4$ wurde nichts abgedunstet, sondern das Digitalysat einfach mit Ringer-Lösung verdünnt.

- Die benutzten Temporarien stammten aus dem Oktober 1913 und waren in meinem frostfreien Keller überwintert worden. Wie immer bei Versuchen im Winter wurden die gewählten Tiere abends in das angewärmte Laboratorium gebracht, wo sie bis zu der etwa 20 Stunden später folgenden Untersuchung blieben. Wegen der Einzelheiten der Technik verweise ich auf frühere Beschreibungen. Um über die Reaktionsstärke der Tiere immer Klarheit zu haben, wurde neben jeder Digitalysatprüfung zur Kontrolle an 4-5 Tieren ein Infus Fol. Dig. titr. $1: 10$ in der Menge von $1 / 50$ F. G. eingespritzt. Die Reaktionen waren im allgemoinen von befriedigender Gleichmässigkeit. Nur bei einigen Untersuchungsreihen zeigten die mit dem Blätterinfus eingespritzten Tiere als Folge zu grosser Erwärmung eine Reaktionszeit, die gegenüber der Norm um etwa $10 \mathrm{pCt}$. zu kurz war; und zwar war dies bei 7 von den 29 Untersuchungsreihen der Fall. In diesen 7 Reihen wurden die Zeiten bei den in der Tabelle 1 mit * bezeichneten Digitalysattieren für die Berechnung um den entsprechenden Prozentsatz erhöht.

Wie man in Tahelle 1 sieht, haben sich Reihen ergeten von ziemlich grosser Regelmässigkeit. Die Tabelle ist ebenso wie die entsprechenden in den früheren Arbeiten hergestellt. Die zu einer Reihe zusammen gehörenden Frösche sind von links nach rechts so geordnet, dass jedesmal das am langsamsten (z. B. in der ersten Reihe mit $91 / 2$ Minuten) reagierende Tier zuerst, das am schnellsten reagierende (hier mit $81 / 2$ Minuten) zuletzt steht. Danach richtet sich die Reihenfolge in den übrigen Kolumnen, sodass die erste Zahl der Herzfrequenzen $(61)$, sowie der Tiergewichte $(27,4)$, der injizierten Flüssigkeitsmengen $(1,0)$ und der Wertquotienten $(2,9)$ dem ersten Tier mit der längsten Reaktionszeit angehört, usw. Die Zeit t wurde immer erst dann aufgezeichnet, wenn die ganze Kammer fest kontrahiert war. Wenn nach einer Stunde keine kräftige Digitaliswirkung erkennbar war, so wurde die Zeit mit $\infty$ notiert. Wenn die Kammer zu dieser Zeit zwar noch nicht stillstand, jedoch eine kräftige Digitaliswirkung aufwies, so wurde dasselbe Zeichen in Klammer gesetzt $(\infty)$. Das Uebrige erklärt sich von selbst. 


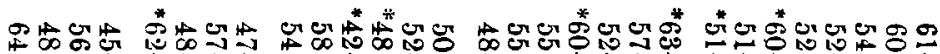

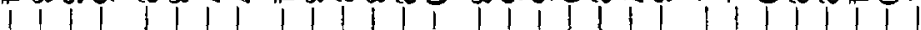

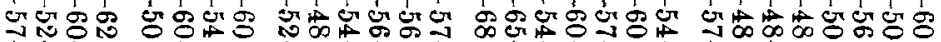

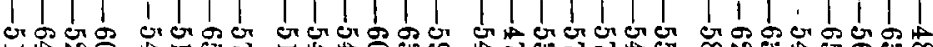

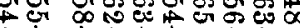

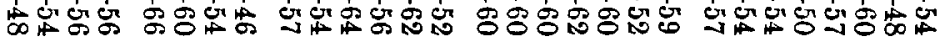
N D.

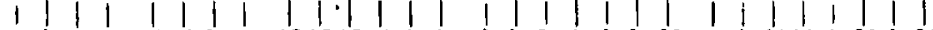





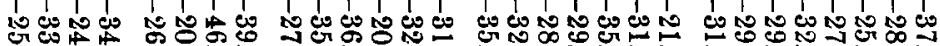

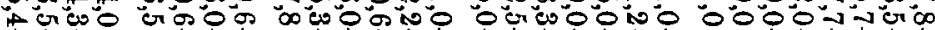

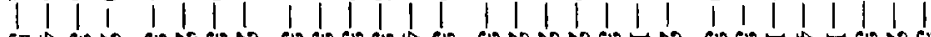

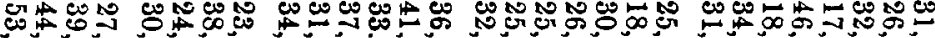

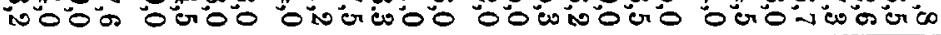



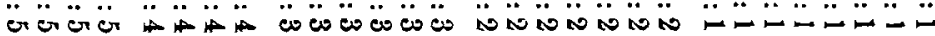


0000005000005000000500000005

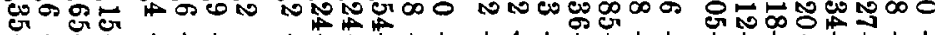

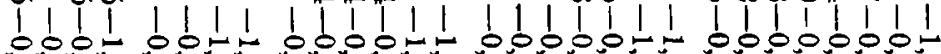



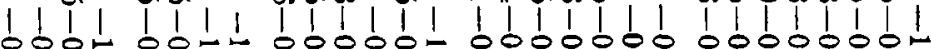

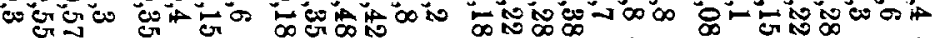

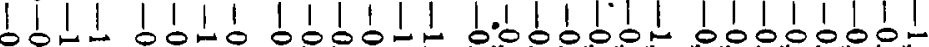
6.0”



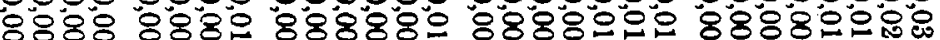

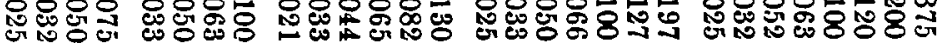

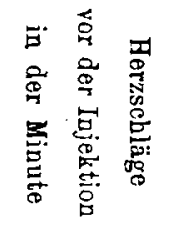

$\|$ 11

Lösungsverhältnis der Flüsssigkeit

relativ zum

Froscli-

gewicht

보ㅇㅝㅗㅇ



g od. cem Digita-

lysat pro Gramm Froschgewicht

ชิ8



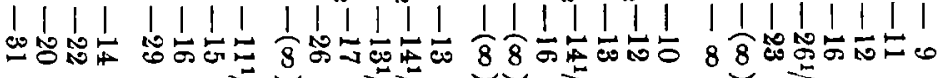
-


西告

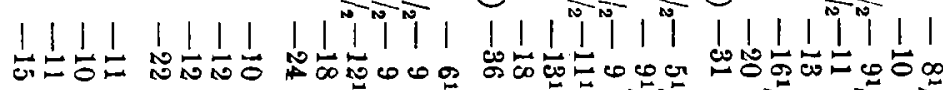

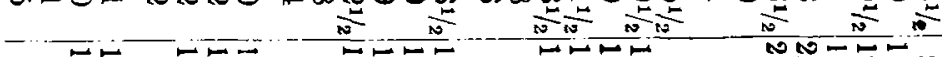

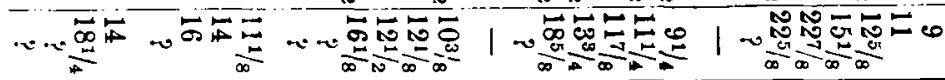

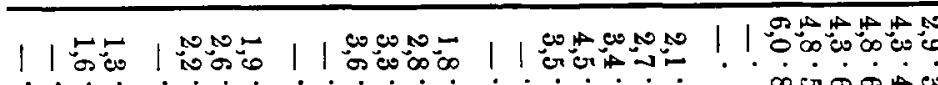

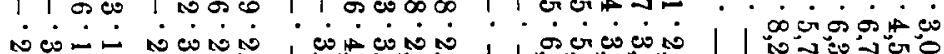

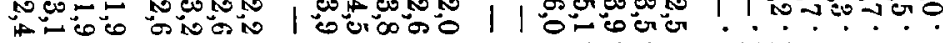

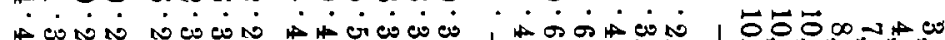
ON ن ن inde . U



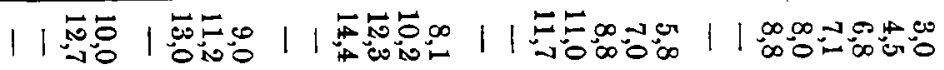


An der Tabelle ist besonders lehrreich die drittletzte Kolumne, die ohne Rücksicht auf die Verdünnung der Injektionsflüssigkeit den Durchschnitt von $\mathrm{v}$ angibt. Es zeigen sich hier wieder dieselben Gesetze, wie bei den früheren Tabellen. Innerhalb jeder Lösungsgruppe (z. B. der Gruppe 1:1) wird $\mathrm{v}$ jedesmal grösser, je kleiner die eingespritzte Flüssigkeitsmenge war. Das bedeutet auch hier wieder: Aus einer kleineren Flüssigkeitsmenge wird ein verhältnismässig grösserer Anteil der vorhandenen Glukoside ausgenützt als aus einer grösseren Flüssigkeitsmenge der gleichen Konzentration. Das geschieht aber natïrlich nicht im gleichen Schritt. Bei einer Verminderung der Dosis von $1 / 25$ auf $1 / 200$, also um das achtfache, wächst der Valor von 3,0 auf 8,8 , d. h. um nicht ganz das dreifache.

Tabelle 2.

Valor je nach Lösungsstärke und Injektionsmenge.

\begin{tabular}{c|c|c|c|c|c}
\hline \multirow{3}{*}{ bei Lösung } & \multicolumn{5}{|c}{ bei Injektion von } \\
\cline { 2 - 6 } & $1 / 25$ & $1 / 40$ & $1 / 50$ & $1 / 85$ (u. 1/80) & $1 / 100$ \\
\cline { 2 - 6 } & \multicolumn{6}{|c|}{ des Frosehgewichtes } \\
\hline $1: 1$ & 3,0 & $?$ & 4,5 & 6,8 & 7,1 \\
$1: 2$ & 2,9 & 3,5 & 4,4 & 5,6 & 5,85 \\
$1: 3$ & 2,7 & 3,4 & 4,1 & 4,8 & - \\
$1: 4$ & 2,25 & 2,8 & 3,25 & 3,25 & - \\
$1: 5$ & 2,0 & 2,55 & - & - & -
\end{tabular}

Ferner: Wenn man die gleich grossen Injektionsmengen der verschiedenen Lösungen zusammenstellt (Tab. 2), so findet sich, dass der Valor niemals im gleichen Verhältnis mit der Verdünnung sinkt. Auch hier zeigt sich, dass ein umso grösserer Teil der Glukoside ausgenützt wird, je schwächer die Lösung war. Hiernach ist es leider unmöglich, bei gleichbleibender Injektionsmenge (z. B. ${ }^{1} / 80$ F. G.), aber bei wechseinder Verdünnung einen Wert mit voller Genauigkeit zu bestimmen. Eine auf diese Weise gegenüber einem Mittelwert als schwächer gefundeno Lösung ist in Wirklichkeit immer noch schwächer als sie gefunden wurde; eine als stärker nachgewiesene ist in Wahrheit immer noch stärker als der Befund es erkennen lässt.

Das sind Ergebnisse, wie sie in diesem Grade nicht bei den Blättern, wohl aber bei den drei bisher untersuchten Reinpräparaten hervorgetreten waren. Am besten vergleicht man das umstehende Diagramm, das auf Grund der letzten Kolumne der Tabelle 1 aufgestellt ist, mit: den ebenso gewonnenen früheren Diagrammen. So 'stark anstcigende Linien wie. hier finden sich nicht bei den Blättern, aber fast genau so beim Gitalin ${ }^{1}$ ). Anf diese auffällige Erscheinung komme ich noch unten zurück.

Auch wenn man die Werte zusammenstellt, die bei gleichen Digitalysatmengen; aber beí wechselnden Lösungen und Flüssigkeits-

1) Diese Zeitschriff, 14. Bd. Taf. XII. Tab. 9 und 14. - Nur die Linie des durch Eindunsten gewonnenen Infuses $1: 6,6$, die für Wertmessungen nicht in Betracht kommt, steigt mässig an. 
Diagramm des Digitalysatum Bürger.



mengen erzielt wurden (Tab. 3), so findet man ein Bild, das mit dem von Gitalin gezeigten ${ }^{1}$ ) eine grosse Aehnlichkeit hat.

Tabelle 3.

\begin{tabular}{|c|c|c|c|c|c|}
\hline \multirow{2}{*}{ 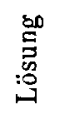 } & \multicolumn{5}{|c|}{ Kubikzentimeter Digitalysat pro Gramm Froschgewicht } \\
\hline & $0,0200-0,0197$ & $0,0130-0,0120$ & 0,0100 & $0,0066-0,0063$ & $0,0052-0,0050$ \\
\hline $\begin{array}{l}1: 1 \\
1: 2 \\
1: 3 \\
1: 4\end{array}$ & $\left.\begin{array}{l}1 / 50=4.5 \\
1 / 25=5,8\end{array}\right\} 5,15$ & $\left.\begin{array}{l}1 / 80=6,8 \\
1 / 40=7,0 \\
1 / 25=8,1\end{array}\right\} 7,3$ & $\left.\begin{array}{l}1 / 100=7,1 \\
1 / 80=8,8 \\
1 / 25=9,0\end{array}\right\}$ & $\left.\mid \begin{array}{l}1 / 150=8,0 \\
1 / 75=11,0 \\
1 / 50=12,3 \\
1 / 40=11,2\end{array}\right\} 10,6$ & $\left.\begin{array}{c}1 / 200=8,8 \\
1 / 100=11,7 \\
1 / 80=13,0 \\
1 / 60=12,7\end{array}\right\} 11,5$ \\
\hline
\end{tabular}

\section{Fragen.}

a) Wie ist künftig die Wertmessung der Digitalis-Dialysate

\section{auszuführen?}

Die im Vorhergehenden mitgeteilten Befunde müssen natürlich auf die künftige Wertmessung des Digitalysats ihren Einfluss ausüben. Da bei der Prüfung am Frosch das Digitalysat den Reinpräparaten nahesteht und weil deren physiologische Stärke nur an ihrer wirksamen Mindestdosis gemessen werden kann, so ist auch für das Digitalysat eine Mindestdosen-Methode nötig. Es fragt sich, wie eine solche mit

1) Diese Zeitschr. 14. Bd. 'Tab. 7 auf S. 283. S.-A. S. 22. 
dem geringsten Aufwand an Mühe, Zeit und Tieren, auch mit Rücksicht auf die Grenzen der hauptsächlich in Betracht kommenden physiologischen Digitalysatwerte, am besten einzurichten ist.

Aus der Tab. 1 geht hervor, dass im Winter die wirksame Mindestdosis bei jeder Verdünnung von $1: 2$ bis zu $1: 4$ gefunden werden kann. Bei 1:4 geht das natürlich nur, wenn das Präparat so stark ist, dass es bei dieser Verdünnung noch genügend wirkt. Im Sommer erfordert die geringere Reaktionsfähigkeit der Tjere eine weniger starke Verdünṇung. Daher kann die Frage nicht mit einem kurzen Schema beantwortet werden. Eine ausführliche Regel aber darüber, welche Verdünnungen und Injektionsmengen im Sommer und Winter die zweckmässigsten sind, kann ich aus meinen bisherigen Versuchen noch nicht ableiten, da deren Zahl noch beschränkt war. Soweit meine Erfahrung reicht, kann ich bis jetzt nur folgendes sagen: Die Feststellung der eigentlichen Mindestdosis ist beim Digitalisdialysat vielleicht noch schwieriger als bei den Reinpräparaten; sie wird nur dadurch erleichtert, dass man die ungefähren Grenzen kennt, innerhalb deren sich die fraglichen Werte bowegen. Oberhalb von 30 Minuten ist die sichere Festlegung eines systolischen Stillstands ausgeschlossen. Man kann aber eine hinreichend genaue Wertmessung gewinnen, wenn bei mögtichst kleinen Dosen aus der gewählten Zahl vou Tieren mindestens die Hälfte unterhalb von 30 Minuten den Stillstand zeigt. Natürlich muss man sich über die Reaktionsfähigkeit der Tiere bei jeder Prüfung vergewissern. Ich benutze hierzu als das Zuverlässigste immer ein an demselben Tage frisch bereitetes Infus Fol. Dig. titr. 1:10, wozu nur 4-5 Tiere nötig sind. Da man mit Rücksicht auf die bekannte Schwankungsbreite bei einer Prüfung gewöhnlich mit acht Tieren für das Dialysat auskommt, so sind im ganzen meistens nur zwölf Tiere nötig, wenn die Reaktionen gleichmässig sind. Es werden immer zwei Tiere für das Dialysat und ein Tier für das Infus nebeneinander auf den Kasten gelegt und genau gleichartig behandelt. Die herauskommenden Werte stehen jedenfalls den wahren Werten sehr nahe. Wenn Endzahlen abgerundet werden müssen, so hat das bei. unternormalen Werten nach unten, bei übernormalen nach oben zu geschehen. Ich hoffe auf eine baldige Gelegenheit, Genaueres über die Prüfungen berichten zu können.

Den gewonnenen Wert mit der Zahl der Mindestdosis selbst zu bezeichnen, z. B. mit ${ } 0,011 \mathrm{ccm}$ auf $1 \mathrm{~g} \mathrm{~F}$. G. ${ }^{*}$, kann ich mich 'nicht entschliessen. Denn die, bei dem jewoilig günstigsten Tier gefundene Mindestdosis unterliegt zu sehr dem Zufall. Wählt man einen Durchschnitt, so ist doch immer die reziproke Zahl, also der Valor, der mit dem Wert selbst steigt und fällt; die viel handlichere Bezeichnung.

Wenn ich ron den nach der neuen Methode am normalen Digitalysat des Handels ausgeführten Prüfungen diejenigen herausziebe, die mit entsprechenden der Tab. 1 vergleichbar sind, so ergibt sich Tab. 4.

Bei der Lösung von 1:2 dürften die Werte für das normale Digitalysat sich zwischen 6,3 und 6,9 bewegen; um für die einzelnen F. G. Mengen die genaueren Ziffern getrennt festzulegen, reichte die Zahl meiner Versuche noch nicht aus. 
Tabelle 4.

Prüfungen im Winter.

\begin{tabular}{|c|c|c|c|c|}
\hline Lösung & F. G. & $\begin{array}{c}\text { Normales } \\
\text { Digitalysat } \\
\mathbf{v}\end{array}$ & $\begin{array}{c}\text { Digitalysat } 1918, \text { IX: } \\
\mathrm{v}\end{array}$ & $\begin{array}{l}\text { Verbättnis } \\
\text { der beiden } \\
\text { Digitalysato } \\
\text { ungefahr }\end{array}$ \\
\hline $\begin{array}{l}1: 3 \\
1: 3 \\
1: 3\end{array}$ & $\begin{array}{l}1 / 10 \\
1 / 80 \\
1 / 80\end{array}$ & $\begin{array}{r}7,5 \\
.7,8 \\
8,7\end{array}$ & $\begin{array}{c}10,2 \\
12,3 \\
\text { (zufolge Interpolation } \\
\text { wahrscheinlich:) } 13,2\end{array}$ & $2: 3$ \\
\hline
\end{tabular}

b) Mit welchem Valor soll künftig das normále Digitalysat bezeichnet worden?

Bisher war das zum ärztlichen Gebrauch bestimmte Digitalysat Bürger mit dem Valor 5,0 bezeichnet worden, weil ich die Prüfungen in den ersten Jahren mit dem unverdünnten Präparat ausgeführt hatte und dabei eben als Normalwert stets ungefähr 5,0 erhielt. Nachdem aber jetzt eine genauere Prüfungsart gefunden ist, die für das normale Digitalysat einen höheren Wert ergibt, so fragt es sich, ob -die frühere Bezeichnung festgehalten werdon soll.

Bei dieser Frage muss die Wertbezeichnung der Fol. Dig. titr. in Betracht gezogen werden. Denn die Fol. Dig. titr., die als die beste Form der Droge die volle Digitaliswirkung bieten und sich im Gleichbleiben ihrer Wirkungsstärke seit langer Zeit bewährt haben, dienen ja seit Jahren nicht nur für das Digitalysat, sondern auch für andere, unter physiologischer Kontrolle stehende Präparate, als Masstab ihrer Wirkungsstärke. Andrerseits braucht $z$ war bei $z$ wei verschiedenartig hergestellten Digitalispräparaten das Verhältnis ihrer klinischen Wirkungsstärken dem Verhältnis ihrer physiologischen Werte nicht zu entsprechen; und daher ist ihr physiologisches Verhältnis auch nicht massgebend fïr ibre Dosierung beim Kranken. Sollte aber zufällig das eine Verhältnis mit dem andern ungefähr übereinstimmen, so wäre dieser Umstand doch wert, bei der Bezeichnung berücksichtigt zu werden.

Die Fol. Dig. titr. ${ }^{1}$ ) zeigen nun an Temporarien im Winter bei Infusen von $1: 15$ bis $1: 20$ mindestens $V=50$, bei den gewöhnlich von mir benutzten Infusen von $1: 10$ durchschnittlich etwa $V=43$, weshalb für das 10 proz. Infus von mir der Wert $V=4,0$ festgehalten worden ist. Da nun das normale Digitalysat zwar bei der Verdünnung von 1:3 den Wert 7,5 bis 8,7 , bei der geringeren Verdünnung von $1: 2$ aber etwa 6,3 bis 6,9 zeigt, so erscheint für das normale Digitalysat als zweckmässigste Bezeichnung seines Mindestwertes $\mathrm{V}=6,0$. Dem würde das klinische Wirkungsverhältnis entsprechen. Beim kranken Menschen kann man von den Fol. Dig. titr. (etwa 0,12 in Pulverform oder) $0,15 \mathrm{im}$ Infus eingenommen hinsichtlich der Wirkung als Aequivalent betrachten mit 1,0 des Digitalysat Bürger. Wenn nun $1,5 \mathrm{~g}$ des 10 proz. Blätterinfuses beim Menschen äquivalent ist mit $1,0 \mathrm{~g}$ des Digitalysats,

1) Diese Zeitschrift, 14. Bd. Tab. B. 
so ist das Verhältnis ihrer Wirkungsstärke umgekehrt, d. h. $=1,0: 1,5$, ebenso wie die physiologischen Werte $=\mathbf{4}, 0: 6,0$.

Nach der Art seiner Herstellung müsste das Digitalysat eigentlich beim Frosch einen noch höheren Wert zeigen. Denn $10 \mathrm{~g}$ des fertigen Präparates sind gewonnen aus $10 \mathrm{~g}$ der frischgrünen Blätter; das ist gleich ungefähr $2 \mathrm{~g}$ der getrockneten Blätter oder physiologisch entsprechend $20 \mathrm{~g}$ des 10 proz. Infus. Fol. Dig. titr. Vom Digitalysat müssten also $10 \mathrm{~g}$ beim Frosch doppelt so stark wirken wie $10 \mathrm{~g}$ des 10 proz. Blätterinfuses; mit anderen Worten: das Digitalysat müsste den doppelten Valor des 10 proz. Infuses, d. h. bei schwacher Verdünnung noch $V=8,0$ zeigen. - Da das Digitalysat etwas weniger stark wirkt, so habe ich anfangs vermutet, dass in dem bei der Dialyse verbleibenden Rückstand ein Teil der spezifischen Bestandteile zurückgehalten werden könnte; und ich habe deshalb in verschiedenen Jahren zweimal diesen Rückstand an Fröschen untersucht. Dabei konnte aber eine deutliche Digitaliswirkung trotz gründlicher Prüfung nicht beobachtet werden. Also kann eine nennenswerte Menge digitalisartig wirkender Substanzen in dem Rückstand nicht enthalten sein. Der Grund dafür, dass der Valor des Digitalysats in Wirklichkeit etwas niedriger gefunden wird, als seiner Herstellung entspricht, dürfte sich aus dem folgenden $A$ bschnitt ergeben.

Jedenfalls muss man dem Obigen zufolgo $\mathrm{V}=6,0$ als die am meisten angemessene Wertbezeichnung des Digitalysats betrachten. Daher wird die herstellende Firma diese Bezeichnung künftig statt des bisherigen $\mathrm{V}=5,0$ anwenden, ohne dass aber die Wirkungsstärke selbst irgendwie verändert wird. Die Dosierung muss demnach ebenfalls genau dieselbe bleiben wie bisher.

c) Warum steht das Digitalysat in seiner Wirkung beim Frosch den Reinpräparaten, besonders dem Gitalin, näher als den Blättern?

Während die Fragen $a$ und $b$ wesentlich praktischer Natur waren, hat die schon im Schluss des Abschnitts I aufgetretene Frage, warum das Digitalysat beim Froseh den Reinpräparaten (besonders dem Gitalin) so viel näher steht als den Blättern, zunächst eine mehr theoretische Bedeutung. Aus den Tabellen hatte sich, wie für das Gitalin, so auch für das Digitalysat das Gesetz ergeben: Die Ausnutzung der vorhandenen wirksamen Substanz aus grösseren in den Froschlymphsack eingespritżten Mengen ist nur etwa halb so gut wie bei etwa viermal kleineren eingespritzten Mengen der gleichen Konzentration, während beim Blätterinfus die Ausnutzung aus den grösseren Injektionsmengen fast ebenso gut ist wie aus den kleineren. Aus den Froschlymphsäcken wird das Digitalysat also verhältnismässig weniger rasch resorbiert als das Blätterinfus. Daraus erklärt sich erstens, dass der Valor des Digitalysats, wenn nicht die dünnste Lösung gebraucht wird, etwas geringer ausfällt als erwartet werden sollte; und zweitens erklärt sich daraus überhaupt die in der Einleitung erwähnte grössere Schwierigkeit bei der Wertprüfung des Digitalysats gegenüber dem Blätterinfus. Infolge dieser Schwierigkeit wird es eben nötig, für das Digitalysat eine Prüfungs- 
methode zu wählen, die der Mindestdosenbestimmung möglichst nahe kommt.

Die F'rage, warum das Digitalysat in seiner Resorption beim Frosch vom Blätterinfưs abweicht, lässt sich bis jetzt fast nur durch Vermutungen beantworten. Das Verhalten der Reinpräparate erklärt sich von selbst dadurch, dass ihnen gewisse nicht-spezifische Stoffe fehlen, die in den Blättern vorhanden sind und bei der Resorption des Infuses mithelfen. Fs bleibt nun kaum etwas anderes übrig, als anzunehmen, dass von diesen nichtspezifischen Stoffen ein Teil auch dem Digitalysat fehlt, also bei der Dialyse zurückgehalten wird. Es müsste sich dann jedenfalls um einen kolloidalen Stoff handeln, was ja sehr gut möglichr ist. Ernst Oppenheimer hat aus dem pharmakologischen Institut Freiburg i. B. mitgeteilt, dass Digitoxin und Gitalin durch Dialysierhülsen glatt durchgehen, Saponin aber nicht ${ }^{1}$ ).

Ein Nachteil für die klinische Anwendung des Digitalysats folgt aus seiner langsameren Resorbierbarkeit beim Frosch nicht. Denn durch tausendfache klinische Beobachtung steht es ja fest, wie vorzüglich das Digitalysat vom Magen und rom Unterhautzellgewebe des Menschen resorbiert wird. Dass das Fehlen eines durch die Dialyse entfernten nichtspezifischen Stoffes vielleicht sogar einen Nutzen bringt, wird durch folgende Ueberlegung wahrscheinlich. Bekanntlich verliert eine frisch bereitete Tinctura Digitalis im ersten Jahre etwa 10 pCt. ihres Wirkungswertes. Die Tinktur enthält zwar von den wasserlöslichen Bestandteilen weniger als ein Blätterinfus; aber die resorptionsfördernden Bestandteile sind in ihr gewiss vorhanden, da aus den Froschlymphsäcken die alkoholarm gemachte Tinktur gerade so gut reşorbiert wird wie das Infus. Nun werden höchstwahrscheinlich die spezifischen Stoffe in der Tinktur und im Fluidextrakt gerade durch die nichtspezifischen, kolloidalen, zum Tei] als Enzyme wirkenden Bestandteile abgebaut. Dies kann nicht geschehen, wenn das betreffende Enzym fehlt. Wahrscheinlich ist das kolloidale Enzym, das in den Blättern die Resorption beschleunigt, dasselbe, das in der Tinktur später die Wertminderung herbeiführt. Durch sein Fehlen im Digitalysat wäre eine Erklärung gegeben dafür, dass bei $\mathrm{ihm}$, solange daraufhin geprüft wurde (d. h. sieben Jahre lang), eine zeitliche Verminderung nicht gefunden werden konnte, kurz - für die bisher unverständ̨liche Tatsache der hohen Wertbeständigkeit des Digitalysats.

\section{Zusammenfassung.}

Die Durchprüfung des deutschen Digitalis-Dialysats (Digitalysat Bürger) an Temporarien hat Folgendes ergeben:

1. Je schwächer die Lösung des Digitalysats oder je kleiner die eingespritzte Flüssigkeitsmenge war, um so besser wurden die darin vorhandenen Glukoside aufgesaugt und ausgenützt. In dieser Hinsicht steht das Digitalysat den bisher ebenso untersuchten Glukosiden (Gitalin, Digitalinum verum, g-Strophanthin) näher als den titrirten Blättern.

1) Zur Frage der Fixation der Digitaliskörper im tierischen Organismus und besonders deren Verhalten zum Blut. Zeitschr. f. Bioohemie. 1913. Bd. 55. S. 136. 
2. Wegen dieses Verhaltens ist beim Digitalysat die für die Blätter zweckmässigste einfachere Art der Wertmessung nicht ausreichend. Es ist eine Prüfungsart nötig, die der Mindestdosen-Methode ähnelt.

3. Da bei einer solchen Prüfung am normalen Digitalysat mindestens der Wert $V=6,0$ gefunden wird, so soll das Digitalysat künftig mit dieser Wertbezeichnung versehen werden. Das entspricht auch dem Stärkeverhältnis, in welchem seine Wirkung beim kranken Menschen zu der des 10 proz. Infuses der titrierten Blätter $(V=4,0)$ steht.

4. Der Grund dafür, dass das Digitalysat in seiner Aufsaugungsart den Reinpräparaten näher steht als den Blättern, ist darin zu erblicken, dass bei seiner Herstellung durch Dialyse wahrscheinlich ein kolloider (spezifisch unwirksamer) Stoff entfernt wird, der im Blätterinfus die Aufsaugung beschleunigt. Dass dieser wahrscheinlich zu den Enzymen gehörende Stoff im Digitalysat fehlt, ist umgekehrt vermutlich der Grund für die auffallende Wertbeständigkeit, worin sich das Digitalysat vor den anderen galenischen flüssigen Digitalispräparaten auszeichnet. 